gleichen Winkeln sich kreuzen oder durchflechten, so dass diese Züge rautenförmige Felder umgrenzen, in welchen bei gleichbleibender Tubusstellung blasser erscheinende Hornhautkörperreihen liegen (Fig. 3.).

\title{
Erklärung der Abbildung.
}

Taf. VI.

Fig. 2-3. Hornhautoberfläche eines $1 \frac{1}{2}$ jäbrigen Knaben, H. Wallbracht, aus Giessen. Rohes, auf dem Objecttrïger getrocknetes Präparat. Vergr. 350.

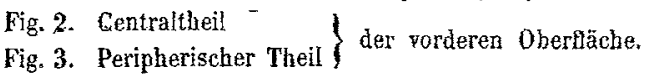

2.

\section{Zur Entwickelungsgeschichte der Corpora amylacea in den Lungen.}

Von Dr. N. Friedreich in Würzburg.

(Hierzu Taf. VI. Fig. 4.)

Schon bei Gelegenbeit meiner ersten Mittheilungen über das Vorkommen amylöser Concretionen in den Lungen hatte ich die. Vermuthung aufgestellt, dass dieselben mit hämorrhagischen Vorgängen im Lungenparenchym in einer näheren genétischen Beziehung stehen möchten (Archiv 1X, 618.). Das Vorkommen der beschriebenen Bildungen einerseits unter Verhältnissen, welche zu Hämorrhagien in das Lungengewebe disponirten, oder welche auf vorhergegangene reichlichere Extravasationen hindeuteten, andererseits das Vorhandensein von Pigmentmassen im Centrum mancher Amylumkörner schien für eine solche Vermuthung hinreichende Anhaltspunkte darzubieten. Neuere Beobachungen aber setzen micl jetzt in den Stand, diese meine früber nur mit einiger Wabrscheinlichkeit ausgesprochene Meinung mit grösserer Bestimmtheit zu wiederholen.

Am 23.Jmi d. J. untersuchte ich nämlich die Lunge eines an lleotyphus verstorbenen 38jährigen Katfmannes, bei welchem neben den gewöhnlichen typhösen Veränderungen des Darms und seiner Drüsen eine ungewöhnlich starke Fettdegeneration des ganżen Herzens, besonders des rechten Ventrikels, sich vorfand. Die Wandungen der Pulmonalarterie zeigten sich, soweit sie makroskopisch verfolgt werden konnten, nicht verändert. Die Lungen beiderseits in ibren unteren Lappen splenisirt, im Uebrigen lufthältig. Auf einem Durchschnitt durch die splenisirten Theile zeigten sich mit der Loape, theilweise auch schon mit blossem Auge zahl- 


\section{8}

reicbe, den interlobulären Bindegewebszïgen folgende, braunrothe oder auch mehr fleischrothe punkiförmige Körperchen, während in den übrigen, lufthältigen Theilen der Lunge Nichts dergleichen entdeckt werden konnte. Die mikroskopische Untersachang zeigte bald braunrothe, bald schwarzrothe, granulöse, runđliche klümpchen von sehn variabler Grösse, welche kanm anders denn als capillärc Extravasationen anfgefasst werden konnten, um so mehr, ałs dieselben meist ziemhich genau dem Verlaufe der interlobulären Gefässe fojgten und mitunter so nahe den Gefässen anlagen, dass man auf den ersten Blick capilläre Aneurysmen vor sich zu haben hätte glauben können. Bald waren diese Klümpchen nackt (Fig. a.), bald aber, und zwar sehr häufig zeigten sich dieselben umkleidet von einer oder auch zwei, seltener drei sehr scharf concentrisch geschichteten Lagen einer mehr durchscheinenden, helleren, gleichmăssig röthlich, gelbröthlich oder auch hellbräunlich gefärbten homogenen Substanz (Fig. b.), welche, wie die chemischen Reactionen wahrscheinlich zu machen schienen, aus dem faserstoffigen, dnrch Blutroth imbibirten Theile des Extravasates gebildet sein mochten. Mitunter schien die centrale Masse, vielleicht durch theilweise Verdunstung ihres Wassergehaltes während des Respirationsactes, derber, trockener und brüchiger geworden zu sein, wie dies wenigstens radiäre, gegen das Centrurn gerichtete 'Sprünge unỏ Einrisse, die sich mitunter auch durch die peripherischen Schichten fortsetzten, andeuteten (Fig. c.). Hie und da zeigte sich auch ein ausgebildeter Hämatoidinkrystall im Innern des centralen Cruorklumpens (Fig. d.). - Zusatz von Essigsäure, mehr noch von Natron machte die Körper im Ganzen, besonders aber die Umhüllungsschichten heller und durchsichtiger, ohne dieselben jedoch zu lösen. Jod ergab die gewöhnliche Reaction auf Proteinsubstanzen. - Im Inbalte der Bronchien liessen die beschriebenen Formationen sich nicht auffinden.

Ueberblicken wir nun alle von mir früher mitgetheilten Thatsachen über die Corpora amylacea in den Lungen und vergleichen wir dieselben mit den eben beschriebenen hämorrahagischen Bildungen, so dürfte es in hohem Grade wahrscheinlich werden, dass jene aus letzteren liervorgehen, dass also nach einem gewissen Zeitraum ihres Bestehens aus den frischen concentrisch geronnenen Extravasationen corpora amylacea geworden wären. Es dürfte demnach del Entwickeluogsgang der letzteren in folgender Weise aufufassen sein: Unter gewissen lrankhaften Zuständen, die entweder in Folge mechanischer Blutstaungen im kleinen Kreislauf (Herzlrankheiten, Verengerungen des Thoraxraums, vgl. Archiv IX. 617; X. 201.), oder an sich schon eine besondere Disposition zu bämorrbagischen Vorgängen mit sich bringen (Typhus), kommt es nicht selten zu capillären Extravasationen in das interlobuläre Bindegewebe der Lungen, deren Gerinnung in der Weise sich gestalten zu können scheint, dass die Blutkörperchen in Form eines runden Cruorklümpchens sich agglomeriren, während der faserstoffige Theil des Extravasates oder vielleicht auch später an derselben Stelle sich wiederholender Extravasationen in concentrischen Lamellen um dasselbe herum sich ablagert. Die weiteren Veränderungen scheinen nun in doppelter Weise sich gestalten zu können. Während nämlich in einem Theil der Körper das in dem Cruorklumpen enthaltene Hämatin sich auflöst und die faserstoffigen Umhüllungssehichten imbibirt und färbt, 
condensirt sich dagegen in einer anderen Reihe von Fällen das Blutroth in der Mitte zu einem krystallinischen oder amorphen körper. So kommt es, dass ir ersteren Falle, da alles Blutroth sich in die umliegenden Gewebe und Flüssigkeiten zertheilt, das Centrum, sowie der Körper überhaupt, sich völlig entfärbt, und nur noch eine granulöse, farblose Masse, welche aus den restirenden Hïllen ảer BlutEörperchen, albuminösen Substanzen a. dgl. bestehen mág, fm Innern zurückbleibt, während in letzteren Falle die früher bescluriebenen Pigmentherne im Centrum des Körpers sich gestalten. Gleichzeitig entwickelt sich durch innere chemische Umsetzungen aus den vorhandenen Proteinstoffen eine der Gruppe der Kohlenhydrate angelörige Substanz, und zwar scheint dies zuerst an den fibrinösen Umhüllungsschichten vor sich zu gehen; wenigstens finden sich, wie ich früber gezeigt habe, nicht selten amylöse Bildungen, deren centraler granulöser Kern durch Jod die gewöhnliche braune Reaction ergibt, während bereits dic peripberischen Schichtungen die blaue Färbung darbieten (Archiv IX. 616.). - Haben sich einmal on einer Stelle des Lungenparenchyms Corpora amylacea in grösserer Zahl entwickelt, so scheinen dieselben als Fremdkörper wirken und durch ihren mechanischen Reiz zu entzünd̆lichen Prozessen Veranlassung geben zu können (Archiv IX. 617.), so dass vielleicht manche Pneunonien in dieser Weise ilive ätiologische Begründung finden dürften.

Schliesslich will ich noch hinzufügen, dass ich vor kurzem in den lungen einer 35jahrigen Frau Concretionen gefunden habe, die zwar morphologisch mit den Corpora amylacea itbereinstimmten, in ibrem chemisclen Verhalten dagegen, namentlich gegen Jod, sich wesentlich differenter Natur ergaben. Die Kranke war gleichfalls an lleotyphus verstorben und zèigte neben gleichzeitiger beträchtiicher Fettdegeneration des Herzfleisches zahlreiche kleine Ecchymosen in der äusseren Haut, sowie in der Schleimhant des Magens und Dünndarms. Diè Lungen zeigten beiderseits in ihren hinteren und unteren Theilen eine ausgedehnte hyperämische Atelectase, welche stellenweise von den kleinsten Formen frischer katarrhalischer, lobulärer Inflitrationen durchsetzt war. Das Mikroskop zeigte in den atelectatischen Partien ziemlich bäufig theils ganz homogene, theils concentrisch geschichtete, rundliche oder ovale Concretionen, von bald melu wasserhellem, bald mehr gelblich waehsartigem, colloidem Schimmer. Hie und da zeigten diese körper eine centrale granulöse Masse, wie frither beschrieben, oder rom Centrum ausgeliende Sprünge and Zerklüftungen, niemàs aber fand jch hier einen Körper, der eine centrale Pigmentmasse dargeboten bätte. Die Grösse derselben war im Durchschnitt etwas kleiner, als die der früher von mir beschriebenen Corpora amylacea. Durch Zusatz von Säuren $\left(\overline{\mathrm{A}}, \mathrm{NO} \mathrm{O}^{5}, \mathrm{ClH}\right)$, selbst von concentrirter $\mathrm{SO}^{3}$ veränderten sich die Körper nicht, auch Aether schien bei der mikrochemischen Reaction dieselben nicht merklich anzugreifen; nur NaO schien sie etwas beller und dmohscheinender zu machen. Dagegen ergab Jodzusatz eine gelbrothe, ziegelrothe, auch braunrothe Reaction in ähnlicher Weise, wie sie Meckel für einen Theil seiner Speckstoffe als ,Jodroth" bezeichnet; jedoch zeigte sie sich von letzterem dadtrich unterschieden, dass, wăhrend Meckel (Charité-Annalen 4. Jahrgang 1853. S. 272.) angibt, dass starke Säuren die jodrothe Farbe zerstörten, gerade im Gegentheil 
dieselbe bei den in Bede stehenden Concretionen nach $Z_{\text {lisatz }}$ einer starken $\mathrm{SO}^{3}$ nutoch schöner und prächtiger hervortrat. - Bei der grossen morphologischen Aênlichkeit, mit der diese letzterwähnten Concretionen mit den eigentlichen amylösen Bildungen in den Lungen- ïbereinstimmten, glaube ich nicht zu irren, wenn ich dieselben in gleiche Reihe mit letzteren setze und die Verschiedenheiten in der chemischen Reaction durch die Annahme einer Verschiedenheit im Aiter oder in der inneren chemischen Entwickelungsstufe derselben exliäre.

\title{
3.
}

\section{Fall von Pneumonomycosis aspergillina.}

\author{
Von Dr. N. Friedreich in Würzburg.
}

(Hierzu Taf. VI. Fig. 5.)

Bei dem gesteigerten Interesse, welches den im Menschen vorkommenden pflanzlichen Parasyten durch Virchow's neuerliche Arbeit (Archiv IX. 55\%.) zu Theil geworden, dïrfte, abgesehen schon von der Seltenheit dieser Zustände, die Mittheilung nachstehenden Falles einer Pneumonomycosis aspergillina gerechtfertigt erscheinen.

Am 9. September 1. J. nämlich machte ich die Autopsie einer 46jährigen Tünchnersgattin, welche nach mehrmonatlichen Leiden unter den Erscheinungen einer chronischen Lungenaffection mit Hydrops Tags vorher verschieden war. Schon vor längeren Jahren wurde die Kranke mehrmals an rechtsseitigen Pneumonien behandelt, später auch an einem pleuritischen Exsudate derselben Seite, wovon sich noch eine mässige Verengerung der rechten Thoraxhälfte mit Dämpfung des Schalles besonders hinten und unten erhalten batte. Später entwickelten sich die Zeichen einer chronischen Bronclitis, zunehmende Athemnoth, Hydrops, starker Livor des Gesichtes und heftiger Venenpuls namentlich an der rechten Halsseite. Als ich die Kranke im Juli d. . mit dem ordinirenden Arzte Hrn. Dr. Carl Reuss zu sehen Gelegenheit hatte, liess sich ausser den letztgenannten Erscheinungen eine rechtsseitige Herzdilatation und hochgradiges Anasarca der unteren Extremitäten mit Ascites erkennen; die Auscultation der Lungen zeigte beiderseits, besonders aber links binten, reichliches Schleimrasseln. Dickeiterige Sputa. In der letzten Woche des August begann unter fieberhafter Steigerung der Hanttemperatur der Hydrops zuzunehmen; etwa 8 Tage vor dem Tode wurden die Sputa copiöser, reichlich mit dunkelrothen, missfarbigen, jedoch nicht fötid riechenden Bestandtheilen untermengt und ziemlich gleichzeitig damit verschwand unter acuter Entwickelung, einer hydropischen Anschwellung des rechten Armes der bisher so intensiv vorhanden gewesene Venenpuls der rechten Halsseite, was auf eine thrombotische Verschliessung der grossen, den rechten Truncus anonymus zusammen- 